03

\title{
Оптический эффект в магнитных эмульсиях при воздействии магнитного поля
}

\author{
(c) С.С. Белых, К.В. Ерин \\ Северо-Кавказский федеральный университет, \\ 355017 Ставрополь, Россия \\ ฯ e-mail: exiton@inbox.ru \\ Поступила в редакцию 18.05.2021 г. \\ В окончательной редакции 28.05.2021 г. \\ Принята к публикации 01.06.2021 г.
}

\begin{abstract}
Исследованы особенности магнитооптического эффекта изменения прозрачности в магнитной эмульсии с низким межфазным натяжением. Произведена интерпретация эффекта на основе приближения аномальной дифракции. Установлены характерные времена воздействия поля, при которых на оптический эффект не влияют процессы коалесценции и структурирования образца. Определено время релаксации эффекта и произведено сопоставление результатов с расчетами по механизмам малых деформаций микрокапель и вращательной броуновской диффузии.
\end{abstract}

Ключевые слова: магнитные эмульсии, изменение прозрачности, аномальная дифракция, деформация микрокапель, ориентированные структуры.

DOI: 10.21883/OS.2021.09.51344.2300-21

\section{Введение}

Магнитные эмульсии (феррожидкостные эмульсии) представляют собой двухфазные системы, состоящие из капель магнитной жидкости, взвешенных в немагнитной несмешивающейся жидкости. Их макроскопическое поведение продиктовано сложными физическими явлениями, происходящими на микроуровне, такими как деформация, объединение и разрушение капель под действием внешних магнитного, электрического или гидродинамического полей. Методы синтеза магниточувствительных эмульсий были предложены на рубеже 70-80-х гг. XX века [1,2]. Первоначально они предназначались для визуализации магнитных записей и контроля качества магнитных головок [3]. В последнее время спектр применения подобных систем существенно расширился за счет развития микрофлюидики $[4,5]$ и биомедицинских технологий [6-8]. С использованием микрокапель магнитных жидкостей предложены методы измерения вязкости и поверхностного натяжения жидкостей $[9,10]$ и даже определения содержания метилового спирта [11]. Традиционно магнитные эмульсии разделяют на две группы по величине межфазного натяжения [12]. Так, в эмульсиях с высоким коэффициентом межфазного натяжения (к таким эмульсиям, как правило, относят среды с взвешенными в воде каплями магнитной жидкости на углеводородной основе) микрокапли в магнитном поле практически не меняют форму, и выстраиваются цепочки вдоль направления линий напряженности. Многократные исследования оптических явлений в дисперсных системах, содержащих микрокапли магнитной жидкости, в основном опирались на весьма выраженные эффекты агрегирования в маг- нитных эмульсиях, приводящие к образованию структур различного размера [13-15]. В работах [16-20] описаны эффекты дифракции и изменения прозрачности в магнитных эмульсиях и подобных им магнитореологических жидкостях, т.е. коллоидах со сравнительно крупными магнитными частицами микронных размеров. Особенностью другого типа магнитных эмульсий с низким межфазным натяжением является образование системы вытянутых вдоль направления поля эллипсоидальных микрокапель [21]. Разумно предположить, что магнитная эмульсия под действием поля по оптическим свойствам может быть подобна дисперсным системам с полностью ориентированными частицами. Теории ориентационных оптических эффектов в дисперсных системах подробно разработаны [22,23]. При этом, в отличие от известных дисперсных систем, в том числе биологического происхождения, в магнитных эмульсиях степенью вытянутости микрокапель можно управлять действием магнитного поля, не изменяя при этом их насыщенной ориентации, а при нулевом поле переходить к описанию системы как совокупности сферических микрокапель. Другой особенностью магнитных эмульсий является чрезвычайная чувствительность к магнитному полю даже самой малой напряжённости. Магнитооптические эффекты в магнитных жидкостях (концентрированных и разбавленных) исследовались ранее, но при этом интенсивность оптического отклика на действие магнитного поля умеренной и низкой напряженности в них, как правило, невелика [24,25]. В магнитных эмульсиях возможно надежное наблюдение магнитооптических эффектов уже при напряженностях магнитного поля 300-500 A/m, что примерно в 5-10 раз меньше, чем в разбавленных магнитных жидкостях [21,25]. Таким 
образом, оптические свойства магнитных эмульсий представляют существенный интерес как с точки зрения всестороннего изучения механизмов их возникновения, так и развития практических применений. Настоящая работа является продолжением исследований, начатых в [21], и мы представляем в ней результаты исследования особенностей оптического эффекта изменения прозрачности в магнитных эмульсиях с малым межфазным натяжением.

\section{Эксперимент}

Для исследований прозрачности в магнитных эмульсиях с низким межфазным натяжением использовалась классическая установка для магнитооптических измерений, аналогичная описанной в [21]. В качестве источника света использовался гелий-неоновый лазер ГН-2П с длиной волны $633 \mathrm{~nm}$, свет которого проходил через стеклянную кювету с образцом, находящуюся в области однородного поля катушек Гельмгольца. Регистрация оптического сигнала производилась при помощи фотоумножителя ФЭУ-27 и цифрового осциллографа AURIS B-423. Для уменьшения разброса экспериментальных данных производились многократные измерения с последующим усреднением результатов.

Эмульсии для экспериментальных исследований синтезировались с использованием магнитной жидкости типа магнетит в керосине с объемной концентрацией твердой фазы 10\% (производитель - НИПИ Газпереработки, г. Краснодар). Эмульсия магнитной жидкости в гидравлическом масле АМГ-10 изготавливалась путем продавливания магнитной жидкости через тонкий капилляр и последующего механического перемешивания. Стабильность системы в значительной степени достигается за счет вязкости масла и близости значений плотности масла и магнитной жидкости. Объемная концентрация магнитной жидкости в образцах эмульсий составляла от 2 до 7\%, а размер капель находился в пределах $0.5-7 \mu \mathrm{m}$. Данные о распределении капель эмульсии по размерам получены методом динамического рассеяния света на приборе Photocor Complex. Эксперименты показали, что в образцах магнитных эмульсий величина оптического эффекта практически не зависит от состояния поляризации падающего света, поэтому плоскость поляризации падающего света была ориентирована под углом $45^{\circ}$ к горизонту.

Магнитное поле создавали при помощи катушек Гельмгольца, помещенных на вращающемся столике так, чтобы вектор напряженности поля мог составлять различные углы с направлением распространения света.

Относительную прозрачность магнитной эмульсии оценивали по значению величины $\delta T=\left(T_{H}-T_{0}\right) / T_{0}$, где $T_{H}=I_{H} / I_{0}^{i}-$ прозрачность системы при воздействии поля и $T_{0}=I_{0} / I_{0}^{i}-$ в его отсутствие соответственно $\left(I_{0}^{i}\right.$ - интенсивность света, падающего на кювету).
Связь между оптической плотностью эмульсии и прозрачностью можно интерпретировать на основе закона Бугера-Ламберта:

$$
T_{0, H}=\exp \left(-2.3 D_{0, H}\right),
$$

где $D_{H}$ и $D_{0}$ - оптические плотности системы в магнитном поле и без него соответственно. В приближении однократного рассеяния оптическую плотность можно выразить через сечение ослабления света частицей $\left(\sigma_{e}\right)$, концентрацию частиц $(N)$ и длину пути света $(l)$ :

$$
D=0.43 \sigma_{e} N l .
$$

Поскольку точное определение числовой концентрации микрокапель в магнитной эмульсии затруднительно, а параметр зависит от нее сложным образом, то для сопоставления результатов эксперимента с оптическими моделями рассчитывалось относительное изменение оптической плотности:

$$
\delta D=\frac{1}{D_{0}} \lg \left(\frac{1}{\delta T+1}\right) .
$$

В работах Н.Г. Хлебцова $[23,26]$ этот параметр является ключевым для описания ориентационного эффекта изменения прозрачности в дисперсных системах (ориентационного турбидиметрического эффекта). Он удобен, в том числе и тем, что при неизменной концентрации частиц определяется только соотношением сечений ослабления света вследствие ориентации частиц полем или, как в нашем случае, вытяжением микрокапель вдоль направления поля:

$$
\delta D=\frac{\sigma_{H}-\sigma_{0}}{\sigma_{0}} .
$$

Параметры $\delta T$ и $\delta D$ имеют противоположные знаки, т.е. при увеличении прозрачности системы в поле $\delta T>0, \delta D<0$, а при уменьшении $-\delta T<0, \delta D>0$.

Воздействие магнитного поля приводит к изменению прозрачности магнитной эмульсии. Знак эффекта зависит от ориентации поля относительно светового луча и среднего размера микрокапель. В импульсном магнитном поле кривая изменения эффекта достаточно типична для электро- и магнитооптических эффектов и состоит из трех областей: нарастание эффекта (I), область насыщения (II) и релаксация после выключения поля (III) (рис. 1). При этом релаксация эффекта для некоторого диапазона размеров частиц может иметь немонотонный характер с переходом значения эффекта через нуль.

Спад эффекта после выключения импульса поля имеет характерные времена релаксации в диапазоне 5-10s. На рис. 2 показаны кривые релаксации эффекта, измеренные после выключения импульса продольного магнитного поля одной и той же напряжённости, но различной длительности. Из кривых видно, что существенного различия во временах релаксации не наблюдается, что 


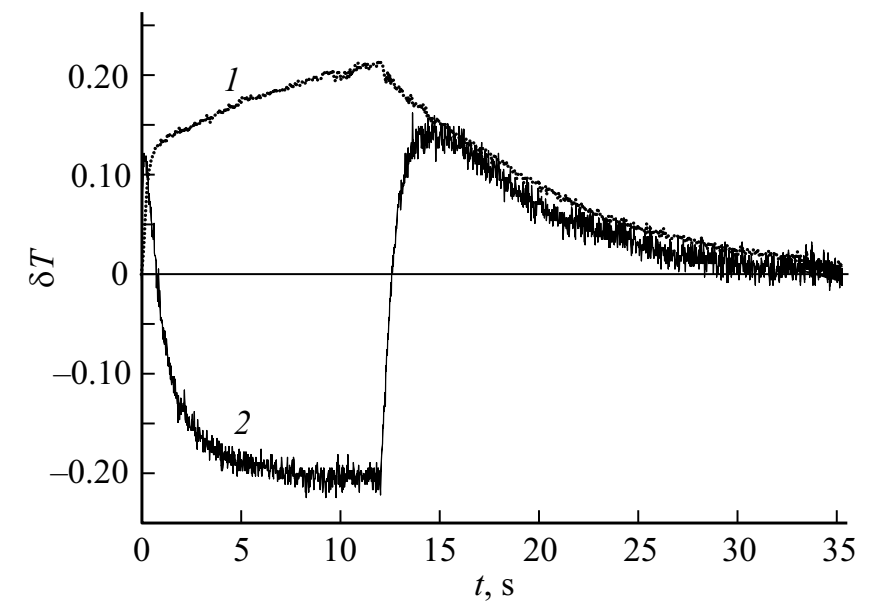

Рис. 1. Кривые магнитооптического эффекта в продольном импульсном магнитном поле длительностью $12 \mathrm{~s}$ и напряженностью $4.5 \mathrm{kA} / \mathrm{m}$ в образцах магнитных эмульсий со средним размером микрокапель 3 (1), $1 \mu \mathrm{m}(2)$.

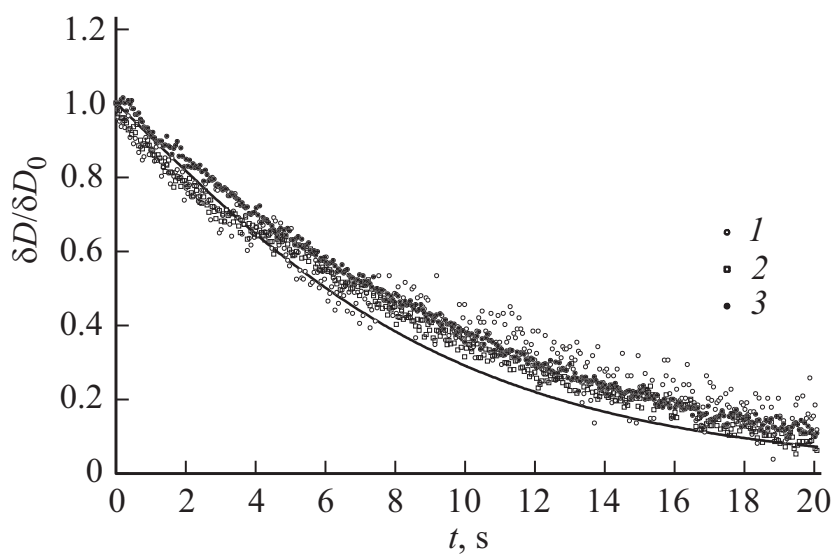

Рис. 2. Кривые релаксации магнитооптического эффекта после выключения импульса магнитного поля длительностью 2 (1), 8 (2), $16 \mathrm{~s}$ (3). Линия - расчет в приближении аномальной дифракции при $\tau=7 \mathrm{~s}$.

может говорить о том, что средний размер микрокапель эмульсии не изменяется при воздействии магнитного поля длительностью до $16-18 \mathrm{~s}$.

Магнитооптический эффект имеет характерную зависимость от ориентации магнитного поля относительно луча света. Для эмульсии с относительно крупными частицами $\left(r_{0}>1.5-2 \mu \mathrm{m}\right)$ знаки эффектов в поле, направленном вдоль луча (в дальнейшем мы будем называть такое поле продольным) и перпендикулярно ему (поперечное поле), имеют разные знаки (рис. 3). В продольном поле прозрачность эмульсии возрастает, а в поперечном снижается. Эффект отсутствует при угле ориентации поля относительно луча света около $40^{\circ}$.

На рис. 4 показана полевая зависимость магнитооптического эффекта, измеренная при подаче линейно возрастающего магнитного поля. Зависимость характеризуется быстрым ростом эффекта при увеличении поля до $1-1.5 \mathrm{kA} / \mathrm{m}$, затем скорость роста существенно замедляется, и эффект переходит в состояние, близкое к насыщению. Было обнаружено, что длительное действие поля с напряженностью выше $4 \mathrm{kA} / \mathrm{m}$ приводит к снижению величины эффекта примерно на 20-25\%. В сравнительно быстро нарастающем поле (за время менее $15 \mathrm{~s}$ ) снижения величины эффекта не наблюдается. Таким образом, можно сделать вывод о том, что влияние процессов коалесценции в магнитных эмульсиях можно существенно снизить, если использовать сравнительно короткие импульсы поля даже достаточно высокой напряженности. Это коррелирует с результатами измерения времени релаксации эффекта, которые демонстрируют одинаковые времена релаксации при длительности воздействия поля до $20 \mathrm{~s}$.

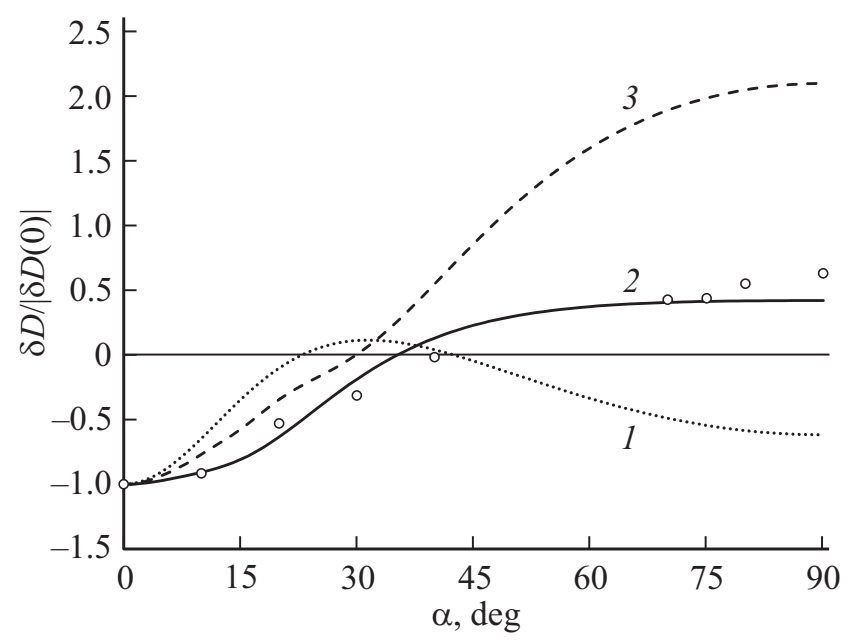

Рис. 3. Зависимость магнитооптического эффекта от угла между направлением поля и луча света. Точки - эксперимент, линии - расчет при $r_{0}=1.5$ (1), 3 (2), $6 \mu \mathrm{m}(3)$.

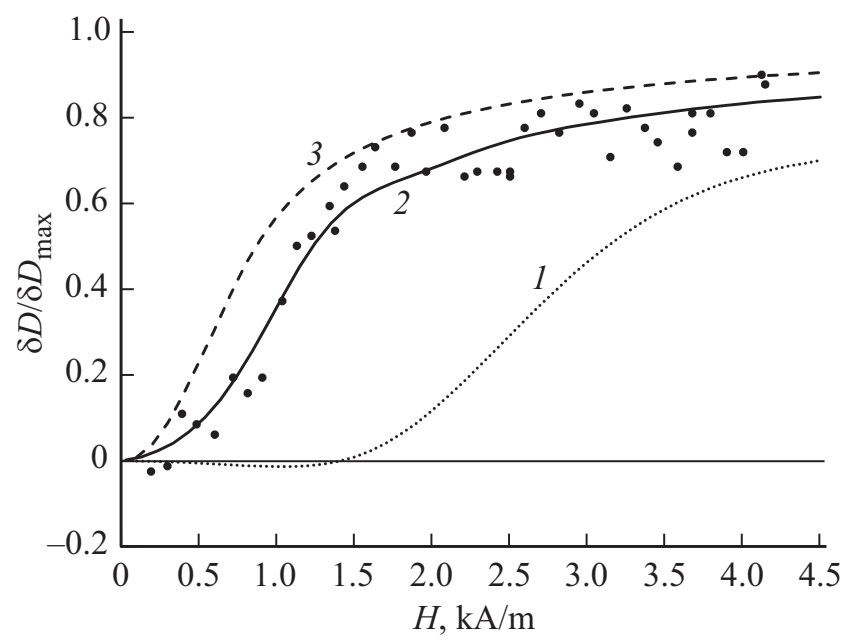

Рис. 4. Полевая зависимость магнитооптического эффекта. Точки - эксперимент; кривые - расчет в приближении аномальной дифракции: $r_{0}=1.5$ (1), 3 (2), $9 \mu \mathrm{m}(3)$. 

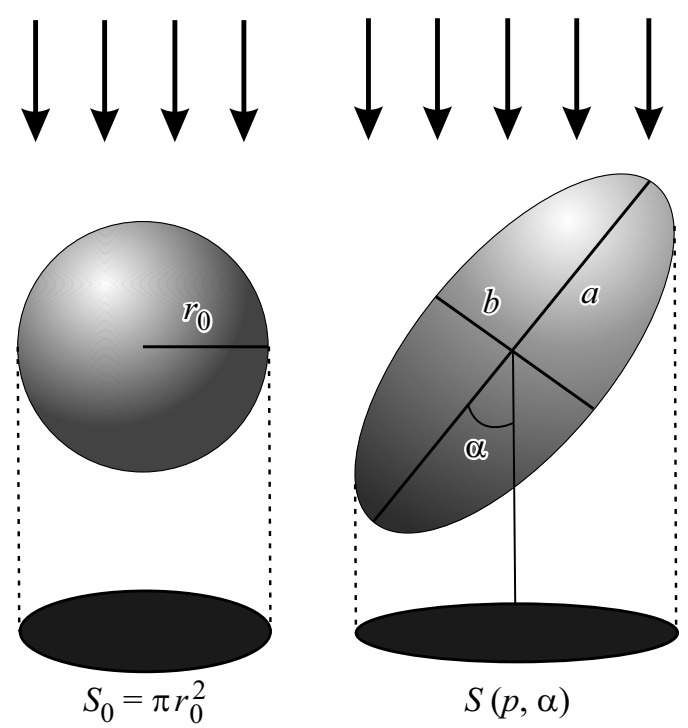

Рис. 5. К расчету сечения ослабления света в приближении аномальной дифракции.

\section{Обсуждение результатов}

Интерпретация оптических эффектов в магнитных эмульсиях возможна с использованием, так называемого приближения аномальной дифракции. Приближение было сформулировано Ван де Хюлстом на основании принципов Гюйгенса и Бабине [26-28] и является обобщением дифракционной формулы Фраунгофера. Оно справедливо для оптически мягких частиц с параметром размера $x=2 \pi r_{0} / \lambda \gg 1$ и с относительным показателем преломления $|m-1| \ll 1$. Оба условия применимы к каплям магнитной эмульсии, так как у исследованных нами образцов $x \approx 10-50$, a $|m-1|=0.06$. Ранее в [21] мы успешно применяли это приближение для качественного описания оптических эффектов для микрокапель магнитной эмульсии.

Рассмотрим ориентацию сфероидальной микрокапли относительно направления падающего света как показано на рис. 5. Сечение ослабления света в приближении аномальной дифракции можно записать как

$$
\sigma_{e}=S(p, \alpha) Q(\rho),
$$

где $S(p, \alpha)$ - площадь тени, зависящая от степени вытянутости микрокапли $(p=a / b)$ и ее ориентации по отношению к свету, а $Q(\rho)$ - фактор эффективности ослабления света. Выражение для $Q(\rho)$ поглощающей частицы (показатель преломления капель использованной нами магнитной жидкости для длины волны $633 \mathrm{~nm}$ составляет $\left.n_{m f}=1.57-0.02 i\right)$ приведено в [27]:

$$
\begin{aligned}
Q(\rho)= & 2-4 \exp (-\rho \operatorname{tg} \beta) \frac{\cos \beta}{\rho}\left(\sin (\rho-\beta)+\frac{\cos \beta}{\rho}\right. \\
& \times \cos (\rho-2 \beta))+4\left(\frac{\cos \beta}{\rho}\right)^{2} \cos 2 \beta .
\end{aligned}
$$

Здесь

$$
\operatorname{tg} \beta=\frac{\operatorname{Im}(m)}{\operatorname{Re}(m)-1} .
$$

Параметр $\rho$ носит название фазового сдвига, и для сфероида, ориентированного под углом $\alpha$ к падающему свету, рассчитывается по формуле:

$$
\rho=\frac{4 \pi r_{0}}{\lambda} \frac{m-1}{\sqrt[3]{p} \sqrt{1+\left(p^{-2}-1\right) \cos ^{2} \alpha}} .
$$

Тогда с учетом (4), (5) и простых геометрических соображений можно получить выражение для сечения ослабления света:

$$
\sigma_{e}=\pi r_{0}^{2} Q(\rho) \sqrt[3]{p} \sqrt{1+\left(p^{-2}-1\right) \cos ^{2} \alpha} .
$$

Как видно из (6) и (7), основными параметрами микрокапли, определяющими величину сечения ослабления, являются соотношение полуосей $p$ и радиус сферы равного объема $r_{0}$. Предполагая, что в процессе деформации объем микрокапли не изменяется, мы можем установить связь между $p$ и $r_{0}$ на основе соотношения, описывающего деформацию капли магнитной жидкости во внешнем магнитном поле напряженностью $H[29,30]$ :

$$
\frac{\mu_{0} H^{2} r_{0}}{2 \gamma}=\left(\chi^{-1}+N_{a}\right)^{2} \sqrt[3]{p}\left(2 p-p^{-2}-1\right),
$$

где $\gamma-$ межфазное натяжение, $\mu_{0}-$ магнитная постоянная, $\chi$ - магнитная восприимчивость материала микрокапли (в нашем случае $\chi=1.4, \gamma=10^{-6} \mathrm{~N} / \mathrm{m}$ ). В использованном нами диапазоне напряженностей поля мы полагаем $\chi=$ const. Размагничивающий фактор вдоль длинной оси $N_{a}$ выражается через соотношение осей сфероида известным соотношением

$$
N_{a}=\frac{1}{2 p^{2}\left(\sqrt{1-p^{-2}}\right)^{3}}\left(\ln \left(\frac{1+\sqrt{1-p^{-2}}}{1-\sqrt{1-p^{-2}}}\right)-2 \sqrt{1-p^{-2}}\right) .
$$

Путем численного решения уравнения (8) можно определить связь между соотношением осей капли и радиусом сферы эквивалентного объема при известных величинах поля и межфазного натяжения. На рис. 4 представлены расчеты зависимостей магнитооптического эффекта на основе формул (3) и (5)-(8). Согласие с экспериментом наблюдается при $r_{0}=3 \mu \mathrm{m}$, что соответствует среднему размеру капель в исследованной в данном случае эмульсии. Обратим внимание на особенность полевой зависимости для микрокапель сравнительно малого размера (около $1.5 \mu \mathrm{m}$ ). Для таких капель полевая зависимость имеет характерный вид с практически горизонтальным участком с малой величиной эффекта вплоть до напряженностей поля $1.5 \mathrm{kA} / \mathrm{m}$, после которого эффект начинает возрастать. Регистрация магнитооптического эффекта с такой особенностью может создать ложное впечатление наличия порогового значения поля, при котором возникает оптический эффект. Подобная 
интерпретация магнитоптических эффектов магнитных коллоидах встречается в литературе [31]. Приближение аномальной дифракции также дает правильное качественное и количественное объяснение зависимости эффекта от угла между направлением магнитного поля и светового луча. В данном случае нами была учтена, в отличие от более ранней работы [21], взаимосвязь между $p$ и $r_{0}$ на основе соотношения (8). Расчеты показывают, что вид зависимости определяется размером исходной микрокапли (рис. 3).

Интерпретация релаксационного процесса после выключения магнитного поля может быть построена на основе теории ориентационных эффектов в дисперсных системах, развитой Н.Г. Хлебцовым [23,26]. Согласно этой теории, релаксация относительного изменения оптической плотности дисперсной системы с произвольным размером частиц описывается в терминах оптических парциальных вкладов, т.е. разложений сечения ослабления света по полиномам Лежандра [23,32]:

$$
\delta D(t)=\sum_{n=1}^{\infty}(4 n+1) f_{2 n} \delta \sigma_{2 n} \exp (-2 n(2 n+1) \Theta t) .
$$

Здесь $f_{2 n}$ - четные коэффициенты разложения ориентационной функции по полиномам Лежандра, $\Theta$ коэффициент вращательной броуновской диффузии, а

$$
\delta \sigma_{2 n}=\pi r_{0}^{2} P_{2 n}(\cos \alpha) \int_{0}^{1} P_{2 n}(z) Q(z) d z / \sigma_{0}
$$

- разложение изменения сечения ослабления света частицы при её ориентации под углом $\alpha$ к падающему свету по полиномам Лежандра (в разложение входят только четные члены, т. е. $n=1$ соответствует полиному второй степени $P_{2}$, а $n=2-$ четвертой $P_{4}$ и т.д.).

Ключевой особенностью выражения (9) является то, что оно предсказывает неэкспоненциальную релаксацию даже для монодисперсной системы частиц из-за учета парциальных вкладов более высокого порядка. Экспоненциальный спад эффекта возможен только в случае рэлеевских частиц ( $\delta \sigma$ мало), а также для более крупных частиц, но только с низкой степенью ориентационной упорядоченности $\left(f_{2 n} \ll 1\right)$. В обоих случаях релаксация ориентационного оптического эффекта характеризуется временем $\tau_{B}=1 / 6 \Theta$. Расчеты по формуле (9), приведенные в [32], показывают, что ориентационные эффекты могут релаксировать как быстрее, так и медленнее простой экспоненциальной зависимости. Общим правилом является сильное искажение экспоненциальной релаксации для таких комбинаций параметров частиц, когда связанный с ориентацией частиц оптический эффект близок к нулю, т. е. величина оптического параметра для ориентированных частиц практически не отличается от таковой для неориентированных. Вторым общим правилом является сильное искажение начальных участков релаксации, а на более поздних стадиях кажущееся время

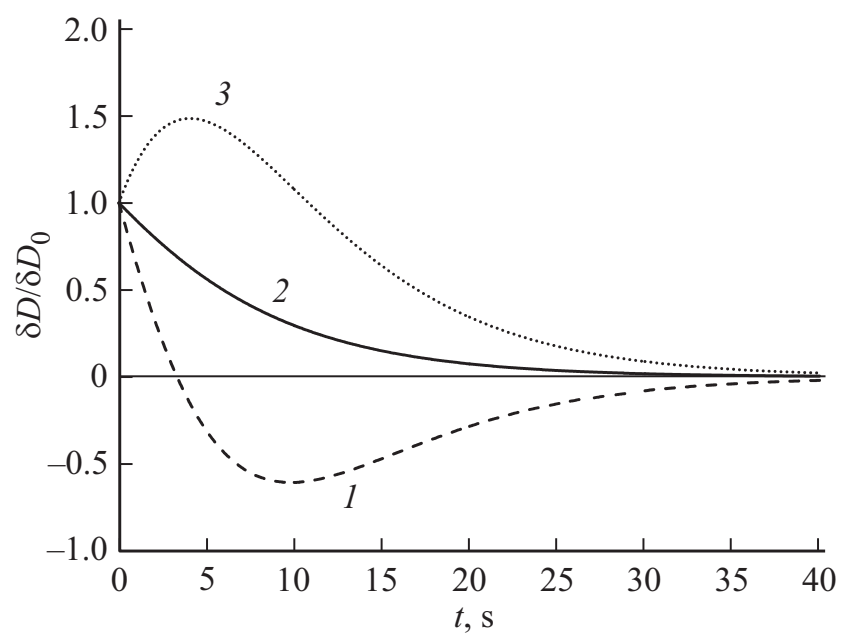

Рис. 6. Расчетные кривые релаксации магнитооптического эффекта для микрокапель с радиусом $r_{0}=1 \mu \mathrm{m}$ после выключения продольного магнитного поля с напряженностью 4.5 (1), $3(2), 1.5 \mathrm{kA} / \mathrm{m}(3)$.

релаксации эффекта близко к истинному. Качественно такой эффект можно видеть на рис. 1.

При использовании теории ориентационных эффектов для магнитных эмульсий необходимо иметь в виду следующее: а) капли эмульсии вытягиваются вдоль направления поля, поэтому в поле всегда присутствует насыщенная степень ориентационной упорядоченности; б) релаксация эффектов происходит одновременно по двум механизмам - вращательная броуновская диффузия и релаксация формы жидкой капли. На рис. 2 представлен расчет кривой спада магнитооптического эффекта в приближении аномальной дифракции с учетом релаксации формы микрокапли с характерным временем около $7 \mathrm{~s}$. Наблюдается удовлетворительное согласие с экспериментальными данными. Отметим, что в зависимости от напряженности поля и его ориентации расчеты предсказывают и немонотонные кривые релаксации эффекта (рис. 6). Это следует из того, что для микрокапель определенных размеров максимальная или минимальная величина оптического эффекта может достигаться не в случае сферической или максимально вытянутой формы микрокапли, а в некотором промежуточном состоянии. Такая немонотонная релаксация действительно наблюдается в эксперименте для образца со сравнительно малым средним размером капель (рис. 1, кривая 2).

Измерение времени вращательной броуновской релаксации для сильно вытянутых частиц широко используется в электрооптических исследованиях для определения размеров и формы макромолекул [33] и других частиц органической и неорганической природы по кривым нарастания или спада электрооптического эффекта (двойного лучепреломления, дихроизма, изменения прозрачности или интенсивности рассеянного света) [34], а также данным динамического рассеяния поляризованного света [35]. Для вытянутых микрокапель 
с радиусом эквиобъемной сферы $r_{0}$, взвешенных в среде с эффективной вязкостью $\eta_{\text {eff, }}$ выражение для времени вращательной броуновской релаксации оптического эффекта можно записать в виде

$$
\tau_{B}=\frac{1}{6 \Theta}=\left(\frac{18 k T}{\pi \eta_{\mathrm{eff}} r_{0}^{3} p^{2}}(\ln (p)+\delta)\right)^{-1} .
$$

Здесь $\delta=-0.662+0.917 p^{-1}-0.05 p^{-2}-$ коэффициент коррекции концевых эффектов.

Описание релаксации формы микрокапель принято строить на основе динамики малых деформаций, сформулированной Тейлором [36] и развитой Маффеттоне и Минале [37]. Согласно этой модели релаксация жидкой капли в вязкой среде к сферической форме происходит с характерным временем [38]

$$
\tau_{d}=\frac{\eta_{m} r_{0}}{\gamma f_{1}}
$$

где $\eta_{m}$ - вязкость среды, окружающей каплю, а безразмерный параметр $f_{1}$ определяется соотношением вязкостей капли и окружающей среды $\tilde{\eta}=\eta_{d} / \eta_{m}$ :

$$
f_{1}=\frac{40(\tilde{\eta}+1)}{(2 \tilde{\eta}+3)(19 \tilde{\eta}+16)} .
$$

Для наших образцов $f_{1} \approx 0.4$, и тогда ожидаемое время релаксации формы капли должно составлять $\tau_{d} \approx 0.1 \mathrm{~s}$. Это более чем на порядок меньше наблюдаемых в эксперименте времен релаксации (рис. 2). При этом использование механизма вращательной диффузии, описываемого формулой (10), также не дает удовлетворительных результатов. Для микрокапель размером $3-5 \mu \mathrm{m}$ с соотношением осей $p=5$ расчеты по формуле (10) дают значения порядка $300 \mathrm{~s}$. Расхождение может быть связано с тем, что формула (11) справедлива для малых деформаций капель, тогда как в эксперименте соотношения осей капель находились в диапазоне $p=3-10$ в зависимости от напряженности поля и, очевидно, не могут считаться малыми. Численное моделирование деформации микрокапель магнитной жидкости в сильных магнитных полях [39] показывает сложный характер зависимости удлинения от времени при воздействии магнитного и гидродинамического полей. Кроме того, на рис. 1 можно видеть, что для эмульсий с заведомо разным средним размером микрокапель, релаксация эффекта происходит с очень близкими временами (за исключением участка сразу после выключения поля). Это также может свидетельствовать в пользу наличия другого механизма релаксации анизотропии, малочувствительного к размеру частицы, что не характерно для теории малых деформаций капель $\left(\tau_{d} \sim r_{0}\right)$ и тем более вращательной броуновской диффузии $\left(\tau_{B} \sim r_{0}^{3}\right)$.

\section{Заключение}

Воздействие магнитного поля на эмульсию магнитной жидкости в минеральном масле АМГ-10 приводит к существенному изменению прозрачности, которое может достигать $25-30 \%$ в поле напряженностью до $5 \mathrm{kA} / \mathrm{m}$. Величина и знак магнитооптического эффекта зависят от напряженности поля и его ориентации относительно луча света. Значительного влияния коалесценции капель под действием поля можно избежать, используя импульсные магнитные поля длительностью не более 15-20s. Удовлетворительная качественная и количественная интерпретация магнитооптического эффекта может быть построена на основе приближения аномальной дифракции света на деформированных микрокапелях эмульсии. Наблюдаемое в эксперименте время релаксации магнитооптического эффекта более чем на порядок превосходит расчеты по известному соотношению, описывающему время релаксации формы жидких капель. Это расхождение может быть связано с существованием более сложного механизма релаксации сильно вытянутых микрокапель, характерное время которого слабо зависит от размера капли.

\section{Финансирование работы}

Работа выполнена при поддержке государственного задания Минобрнауки России на выполнение научноисследовательских работ (проект № 0795-2020-007).

\section{Конфликт интересов}

Авторы заявляют, что у них нет конфликта интересов.

\section{Список литературы}

[1] Romankiw L.T., Briachift N.Y. U.S. Patent 3981844, 1976.

[2] Чеканов В.В., Дроздова В.И. А.С. СССР № 966735, 1982.

[3] Диканский Ю.И. Эффекты взаимодействия частиц и структурно-кинетические процессы в магнитных коллоидах. Дисс. д-ра физ.-мат. наук. Ставрополь, 1999.

[4] Torres-Dıaz I., Rinaldi C. // Soft Matter. 2014. V. 10. P. 8584. doi 10.1039/C4SM01308E

[5] Yang R.-J., Hou H.-H., Wang Y.-N., Fu L.-M. // Sens. Actuators. 2016. V. B 224. P. 1. doi 10.1016/j.snb.2015.10.05

[6] Pankhurst Q.A., Thanh N.T.K., Jones S.K., Dobson J. // J. Phys. D: Appl. Phys. 2009. V. 42. 224001. doi 10.1088/00223727/42/22/224001

[7] Liu Q., Li H., Lam K.Y. // Mol. Pharm. 2018. V. 15. P. 4632. doi 10.1021/acs.molpharmaceut.8b00626

[8] Dailey J.P., Phillips J.P., Li C., Riffle J.S. // J. Magn. Magn. Mater. 1999. V. 194. P. 140. doi 10.1016/S03048853(98)00562-9

[9] Backholm M., Vuckovac M., Schreier J., Latikka M., Hummel M., Linder M.B., Ras R.H.A. // Langmuir. 2017. V. 33. P. 6300. doi 10.1021/acs.langmuir.7b01327

[10] Khokhryakova (Bushueva) C., Kostarev K., Shmyrova A. // Exp. Therm. Fluid Sci. 2019. V. 101. P. 186. doi 10.1016/j.expthermflusci.2018.10.014

[11] Mahendran V., Philip J. // Sens. Actuators B. 2013. V. 185. P. 488. doi 10.1016/j.snb.2013.05.038

[12] Ерин К.В., Белых С.С. // Колл. журн. 2020. Т. 82. № 6. C. 689; Erin K.V., Belykh S.S. // Coll. J. 2020. V. 82. N 6. P. 672. doi 10.1134/S1061933X20060046 
[13] Liu J., Lawrence E.M., Wu A., Ivey M.L., Flores G.A., Javier K., Richard J. // Phys. Rev. Lett. 1995. V. 74 (14). P. 2828. doi 10.1103/PhysRevLett.74.2828

[14] Ivey M., Liu J., Zhu Y., Cutillas S. // Phys. Rev. E. 2000. V. 63. 011403. doi 10.1103/PhysRevE.63.011403

[15] Brojabasi S., Mahendran V., Lahiri B.B., Philip J. // Opt. Comm. 2014. V. 323. P. 54. doi 10.1016/j.optcom.2014.02.041

[16] Horng H.E., Yang S.Y., Tse W.S., Yang H.C., Luo Weili, Hong Chin-Yih // J. Magn. Magn. Mater. 2002. V. 252. P. 104. doi 10.1016/S0304-8853(02)00629-7

[17] Mehta R.V., Patel R., Chudasama B., Upadhyay R.V. // Opt. Lett. 2008. V. 33. N 17. P. 1987. doi 10.1364/OL.33.001987

[18] Philip J., Laskar J.M., Raj B. // Appl. Phys. Lett. 2008. V. 92. P. 221911. doi 10.1063/1.2939100

[19] Martin J.E., Hill K.M., Tigges C.P. // Phys. Rev. E. 1999. V. 59 (5 B). P. 5676. doi 10.1103/physreve.59.5676

[20] Trivedi P., Patel R., Parekh K., Upadhyay R.V., Mehta R.V. // Appl. Opt. 2004. V. 43. P. 3619. doi 10.1364/AO.43.003619

[21] Ерин К.В., Куникин С.А. // Опт. и спектр. 2008. Т. 104. № 2. C. 319; Erin K.V., Kunikin S.A. // Opt. Spectrosc. 2008. V. 104. N 2. P. 2770. doi 10.1134/S0030400X08020227

[22] Электрооптика коллоидов / Под ред. Духина С.С. Киев: Наук. думка, 1977. 200 с.

[23] Khlebtsov N.G., Melnikov A.G., Bogatyrev V.A. // Colloid and Surface A. 1999. V. 148. N 1-2. P. 17. doi 10.1016/S09277757(98)00592-5

[24] Sun J., Yin C., Zhu C., Wang X., Yuan W., Xiao P., Cao Z. // J. Opt. Soc. Am. B. 2012. V. 29. N 4. P. 769. doi 10.1364/josab.29.000769

[25] Ерин К.В. // Опт. и спектр. 2016. Т. 120. № 2. С. 333; Erin K.V. // Opt. Spectrosc. 2016. V. 120. N 2. P. 320. doi 10.1134/S0030400X16020090

[26] Оптическая биомедицинская диагностика. Т. 1 / Перевод под ред. Тучина В.В. М.: Физматлит, 2006. С. 36-82.

[27] Ван де Хюлст Г. Рассеяние света малыми частицами. М.: ИИЛ, 1961. 536 c; Van de Hulst H.C. Light Scattering by Small Particles. N.Y.: John Wiley \& Sons, 1957. 470 p.

[28] Streekstra G.J., Hoekstra A.G., Heethaar R.M. // Appl. Opt. 1994. V. 33. P. 7288. doi 10.1364/AO.33.007288

[29] Afkhami S., Tyler A., Renardy Y., Renardy M., St Pierre T., Woodward R., Riffle J.S. // J. Fluid Mech. 2010. V. 663. P. 358. doi 10.1017/S0022112010003551.

[30] Блум Э.Я., Майоров М.М., Цеберс А.О. Магнитные жидкости. Рига: Зинатне, 1989. 386 с.; Blums E.Ya., Maiorov M.M., Cebers A.O. Magnetic Fluids. Berlin: Walter de Gruyter, 1997. $428 \mathrm{p}$.

[31] Скрипаль А.В., Усанов Д.А. // Письма в ЖТФ. 1997. Т. 23. № 17. C. 7; Skripal' A.V., Usanov D.A. // Technical Phys. Lett. 1997. V. 23. N 9. P. 659. doi $10.1134 / 1.1261847$

[32] Хлебцов Н.Г. Ослабление и рассеяниие света в дисперсных системах с неупо- рядоченными, ориентированными и фрактальными частицами. Автореф. докт. дис. Саратов, 1996; Khlebtsov N.G. Extinction and Scattering of Light in Disperse Systems with Random, Oriented, and Fractal Particles (Theory and Experiment), D.Sci. thesis. Saratov State University, 1996 (in Russian).

[33] Rosenblatt C., Frankel R.B., Blakemore R.P. // Biophys. J. 1985. V. 47. N 3. P. 323. doi 10.1016/S0006-3495(85)83922

[34] Osmolovskaya O.M., Osmolowsky M.G., Petrov M.P., Voitylov A.V., Vojtylov V.V. Colloids and Surfaces A. 2019. V. 586. N 9. 124095. doi 10.1016/j.colsurfa.2019.124095
[35] Pecora R. // J. Nanopart. Res. 2000. V. 2. P. 123. doi 10.1023/A:1010067107182

[36] Taylor G.I. // Proc. Roy. Soc. London, Ser. A. 1934. V. 146. P. 501. doi 10.1098/rspa.1934.0169

[37] Maffettone P.L., Minale M. // J. Non-Newtonian Fluid Mech. 1998. V. 78. V. 227. doi 10.1016/S0377-0257(98)00065-2

[38] Boonen E., van Puyvelde P., Moldenaers P. // J. Rheol. 2010. V. 54. P. 1285. doi 10.1122/1.3490661

[39] Jesus W.C., Roma A.M., Ceniceros H.D. // Commun. Comput. Phys. 2020. V. 24. N 2. P. 332. doi $10.4208 /$ cicp.OA-20170161 